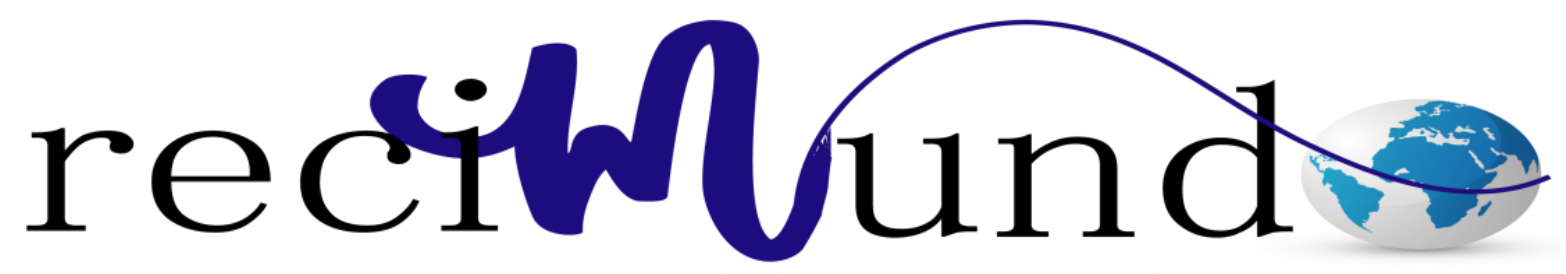

Revista Cientifica Mundo de la Investigación y el Conocimiento

\begin{abstract}
Shirley Mercedes Vite Correa a; Lizandro Nicolás Anzules Cuzco b; Jimmy Eduardo Carreño Ramos ${ }^{\text {c}}$; Mónica Cecilia Burgos Jurado ${ }^{\mathrm{d}}$
\end{abstract}

Terapia intravenosa: cristaloides y soluciones de coloides

Intravenous therapy: crystalloids and colloid solutions

Revista Científica Mundo de la Investigación y el Conocimiento. Vol. 3 núm.3, septiembre, ISSN: 2588-073X, 2019, pp. 1177-1197

DOI: $10.26820 /$ recimundo/3.(3).septiembre.2019.1177-1197

URL: http://recimundo.com/index.php/es/article/view/565

Código UNESCO: 3205 Medicina Interna

Tipo de Investigación: Artículo de Revisión

Editorial Saberes del Conocimiento

Recibido: 15/05/2019

Aceptado: 23/06/2019

Publicado: 30/09/2019

Correspondencia: shirleyvi16co@hotmail.com
a. Médico; Investigador Independiente; Guayaquil, Ecuador; shirleyvi16co@ hotmail.com
b. Médico; Investigador Independiente; Guayaquil, Ecuador; lizandro.anzules@ gmail.com
c. Médico; Investigador Independiente; Guayaquil, Ecuador; dr.jimmycr@ hotmail.com
d. Médico; Investigador Independiente; Guayaquil, Ecuador; monicaburgos_jurado@ hotmail.com 


\section{Terapia intravenosa: cristaloides y soluciones de coloides}

Vol. 3, núm. 3., (2019)

Shirley Mercedes Vite Correa; Lizandro Nicolás Anzules Cuzco; Jimmy Eduardo Carreño Ramos; Mónica Cecilia Burgos Jurado

\section{RESUMEN}

La mayoría de los pacientes de los hospitales reciben algún tipo de tratamiento intravenoso durante su estadía en el hospital, y un número significativo de estos pacientes requieren la reposición de líquidos en forma de expansores de volumen. Hay dos tipos de expansores de volumen, cristaloides y coloides, y cada uno tiene ventajas y desventajas. Comprender el propósito y el uso de cada permitirá a los médicos de la salud para proporcionar a los pacientes el tratamiento más eficaz posible.

Palabras Claves: Terapia Intravenosa; Cristaloides; Coloides; Reposición de Líquido. 


\title{
Terapia intravenosa: cristaloides y soluciones de coloides
}

Vol. 3, núm. 3., (2019)

Shirley Mercedes Vite Correa; Lizandro Nicolás Anzules Cuzco; Jimmy Eduardo Carreño Ramos; Mónica Cecilia Burgos Jurado

\begin{abstract}
Most hospital patients receive some type of intravenous treatment during their hospital stay, and a significant number of these patients require fluid replacement in the form of volume expanders. There are two types of volume expanders, crystalloids and colloids, and each has advantages and disadvantages. Understanding the purpose and use of each will allow health doctors to provide patients with the most effective treatment possible.
\end{abstract}

Key Words: Intravenous Therapy; Crystalloids; Colloids; Liquid Replacement.

\section{Introducción.}




\section{Terapia intravenosa: cristaloides y soluciones de coloides}

Vol. 3, núm. 3., (2019)

Shirley Mercedes Vite Correa; Lizandro Nicolás Anzules Cuzco; Jimmy Eduardo Carreño Ramos; Mónica Cecilia Burgos Jurado

Más de la mitad del peso del cuerpo está compuesto de agua, el líquido esencial para la

vida. El agua dentro del cuerpo se separa en varios segmentos que componen el sistema circulatorio, el líquido que se encuentra que rodea las células, y el líquido dentro de las células. Los fluidos corporales están hechos de combinaciones de agua y electrolitos. Todos estos componentes trabajan juntos para mantener diversas funciones en todo el cuerpo. El objetivo de la gestión de fluidos es la homeostasis, en el que hay un equilibrio en la concentración de solutos que se encuentran en el fluido del sistema circulatorio y el fluido en las células. A veces, enfermedad o lesión provoca este proceso a salir de equilibrio y la terapia de fluidos por vía intravenosa puede ser necesario.

La terapia intravenosa implica fluidos que administran, medicamentos, nutrientes y electrolitos a través de las venas. Soluciones intravenosas se componen de fluido, también llamado el disolvente, y los elementos dentro del fluido, que también se llaman solutos. La terapia intravenosa puede utilizarse para corregir los desequilibrios de electrolitos, lo que aumenta el volumen de fluido en el sistema circulatorio, o sustitución de fluidos que se pierden por enfermedad o lesión. El tipo de fluido dado depende de equilibrio de estado y de fluido del paciente entre el intravascular y los fluidos intracelulares (Bachur \& Shaw, 2015)

Debido a que la administración de fluidos intravasculares prescritos es una práctica muy común en la atención aguda, los médicos de salud deben ser conscientes de los tipos apropiados de fluido para administrar y entender las recomendaciones para el cuidado. Los médicos que atienden directamente a los pacientes tienen un papel importante colaborar y comunicarse con todos los miembros del equipo de salud interdisciplinario, incluyendo farmacia, sobre las mejores prácticas para administrar líquidos por vía intravenosa. 


\section{Terapia intravenosa: cristaloides y soluciones de coloides}

Vol. 3, núm. 3., (2019)

Shirley Mercedes Vite Correa; Lizandro Nicolás Anzules Cuzco; Jimmy Eduardo Carreño Ramos; Mónica Cecilia Burgos Jurado

Los expansores de volumen son tipos de fluidos IV que se utilizan para aumentar la cantidad de fluido en el sistema circulatorio. Se les da cuando la cantidad de líquido en el espacio intravascular es baja, una condición conocida como hipovolemia. expansores de volumen funcionan desplazando el fluido de la solución que rodea las células en el sistema circulatorio. De esta manera, se incrementa el volumen de circulación.

\section{Metodología.}

Para el desarrollo de este proceso investigativo, se plantea como metodología la encaminada hacia una orientación científica particular que se encuentra determinada por la necesidad de indagar en forma precisa y coherente una situación, en tal sentido (Davila, 2015) define la metodología "como aquellos pasos previos que son seleccionados por el investigador para lograr resultados favorables que le ayuden a plantear nuevas ideas".(p.66)

Lo citado por el autor, lleva a entender que el desarrollo de la acción investigativa busca simplemente coordinar acciones enmarcadas en una revisión bibliográfica con el fin de complementar ideas previas relacionadas al uso de cristaloides y soluciones coloides como terapias intravenosas a través de una revisión de literatura, para así finalmente elaborar un cuerpo de consideraciones generales que ayuden a ampliar el interés propuesto.

Tipo de Investigación 


\section{Terapia intravenosa: cristaloides y soluciones de coloides}

Vol. 3, núm. 3., (2019)

Shirley Mercedes Vite Correa; Lizandro Nicolás Anzules Cuzco; Jimmy Eduardo Carreño Ramos; Mónica Cecilia Burgos Jurado

Dentro de toda práctica investigativa, se precisan acciones de carácter metodológico mediante las cuales, se logra conocer y proyectar los eventos posibles que la determinan, así como las características que hacen del acto científico un proceso interactivo ajustado a una realidad posible de ser interpretada. En este sentido, se puede decir, que la presente investigación corresponde al tipo documental, definido por Castro (2016), "se ocupa del estudio de problemas planteados a nivel teórico, la información requerida para abordarlos se encuentra básicamente en materiales impresos, audiovisuales y /o electrónicos". (p.41).

En consideración a esta definición, la orientación metodológica permitió la oportunidad de cumplir con una serie de actividades inherentes a la revisión y lectura de diversos documentos donde se encontraron ideas explicitas relacionadas con los tópicos encargados de identificar a cada característica insertada en el estudio. Por lo tanto, se realizaron continuas interpretaciones con el claro propósito de revisar aquellas apreciaciones o investigaciones propuestas por diferentes investigadores relacionadas con el tema de interés, para luego dar la respectiva argumentación a los planteamientos, en función a las necesidades encontradas en la indagación.

\section{Fuentes Documentales}

El análisis correspondiente a las características que predomina en el tema seleccionado, llevan a incluir diferentes fuentes documentales encargadas de darle el respectivo apoyo y en ese sentido cumplir con la valoración de los hechos a fin de generar nuevos criterios que sirven de referencia a otros procesos investigativos. Para (CASTRO, 2016) las fuentes documentales incorporadas en la investigación documental o bibliográfica, "representa la suma de materiales sistemáticos que son revisados en forma rigurosa y profunda para llegar a un análisis del 


\section{Terapia intravenosa: cristaloides y soluciones de coloides}

Vol. 3, núm. 3., (2019)

Shirley Mercedes Vite Correa; Lizandro Nicolás Anzules Cuzco; Jimmy Eduardo Carreño Ramos; Mónica Cecilia Burgos Jurado

fenómeno". (p.41). Por lo tanto, se procedió a cumplir con la realización de una lectura previa determinada para encontrar aquellos aspectos estrechamente vinculados con el tema, con el fin de explicar mediante un desarrollo las respectivas apreciaciones generales de importancia.

\section{Técnicas para la Recolección de la Información}

La conducción de la investigación para ser realizada en función a las particularidades que determinan a los estudios documentales, tiene como fin el desarrollo de un conjunto de acciones encargadas de llevar a la selección de técnicas estrechamente vinculadas con las características del estudio. En tal sentido, (Bolívar, 2015), refiere, que es "una técnica particular para aportar ayuda a los procedimientos de selección de las ideas primarias y secundarias”. (p. 71).

Por ello, se procedió a la utilización del subrayado, resúmenes, fichaje, como parte básica para la revisión y selección de los documentos que presentan el contenido teórico. Es decir, que mediante la aplicación de estas técnicas se pudo llegar a recoger informaciones en cuanto a la revisión bibliográfica de los diversos elementos encargados de orientar el proceso de investigación. Tal como lo expresa, (Bolivar, 2015) "las técnicas documentales proporcionan las herramientas esenciales y determinantes para responder a los objetivos formulados y llegar a resultados efectivos" (p. 58). Es decir, para responder con eficiencia a las necesidades investigativas, se introdujeron como técnica de recolección el método inductivo, que hizo posible llevar a cabo una valoración de los hechos de forma particular para llegar a la explicación desde una visión general.

Asimismo, se emplearon las técnicas de análisis de información para la realización de la investigación que fue ejecutada bajo la dinámica de aplicar diversos elementos encargados de 


\section{Terapia intravenosa: cristaloides y soluciones de coloides}

Vol. 3, núm. 3., (2019)

Shirley Mercedes Vite Correa; Lizandro Nicolás Anzules Cuzco; Jimmy Eduardo Carreño Ramos; Mónica Cecilia Burgos Jurado

determinar el camino a recorrer por el estudio, según, (Bolívar, 2015) las técnicas de procesamiento de datos en los estudios documentales "son las encargadas de ofrecer al investigador la visión o pasos que debe cumplir durante su ejercicio, cada una de ellas debe estar en correspondencia con el nivel a emplear" (p. 123). Esto indica, que para llevar a cabo el procesamiento de los datos obtenidos una vez aplicado las técnicas seleccionadas, tales como: fichas de resumen, textual, registros descriptivos entre otros, los mismos se deben ajustar al nivel que ha sido seleccionado.

\section{Resultados.}

\section{Sistema circulatorio}

El sistema circulatorio es imprescindible para la distribución de la sangre oxigenada a los órganos y tejidos en el cuerpo. El ciclo cardíaco es el proceso de cada contracción y relajación del corazón que bombea sangre. La transferencia continua de la sangre requiere que los vasos del sistema circulatorio para transportar la sangre que contiene oxígeno a los tejidos del cuerpo. Adicionalmente, la sangre que contiene dióxido de carbono es transportado de nuevo al corazón para ser intercambiado por más oxígeno a través del sistema respiratorio. La sangre también se mueve nutrientes del sistema digestivo en los tejidos y transfiere los productos de desecho y toxinas a los riñones para el filtrado (Bachur \& Shaw, 2015).

Cuando se produce hipovolemia, hay menos volumen en el espacio intravascular, lo que puede conducir a una mala gasto cardíaco con cada ciclo cardiaco. La disminución en la cantidad de sangre bombeada desde el corazón con cada latido del corazón resultados en la perfusión disminuida a los tejidos del cuerpo. Si la hipovolemia se vuelve lo suficientemente grave, el 


\section{Terapia intravenosa: cristaloides y soluciones de coloides}

Vol. 3, núm. 3., (2019)

Shirley Mercedes Vite Correa; Lizandro Nicolás Anzules Cuzco; Jimmy Eduardo Carreño Ramos; Mónica Cecilia Burgos Jurado

cuerpo comienza a compensar a través de la vasoconstricción. Los vasos sanguíneos se hacen más pequeñas con el fin de mantener la presión sanguínea a pesar del volumen disminuida de fluido (Moya, 2013).

La vasoconstricción significativa como un mecanismo de compensación en última instancia puede afectar el flujo de sangre y puede disminuir la circulación. Proporcionar expansores de volumen aumentará el gasto cardíaco debido a que el agregado de fluido al sistema circulatorio aumenta la precarga del corazón, que es la cantidad de sangre que entra en el corazón para ser bombeada por los ventrículos (Siparsky, 2017).

El líquido en el cuerpo se divide en líquido intracelular y extracelular. Fluido intracelular es la que se encuentra dentro de los diferentes tipos de células, mientras que el fluido extracelular es dividen además en dos categorías diferentes conocidos como intersticial y intravascular. El fluido intersticial es la que rodea las células, mientras que el fluido intravascular se encuentra dentro del sistema circulatorio. El fluido intravascular que lleva las células de la sangre también se llama plasma.

El cuerpo mantiene la homeostasis de líquidos y electrolitos entre el intracelular, intersticial, y los espacios intravasculares a través del proceso de ósmosis. La ósmosis es cómo el agua se mueve a través de una membrana semi-permeable, de una zona con una menor concentración de solutos a un área que tiene una mayor concentración de solutos. Las membranas semi-permeables en esta discusión incluyen paredes de los vasos y las paredes celulares. Estas membranas sólo permiten una cierta cantidad de fluido y solutos pase a través. Cuánta ósmosis se produce depende de la presión osmótica, que es la presión que se mueve 


\section{Terapia intravenosa: cristaloides y soluciones de coloides}

Vol. 3, núm. 3., (2019)

Shirley Mercedes Vite Correa; Lizandro Nicolás Anzules Cuzco; Jimmy Eduardo Carreño Ramos; Mónica Cecilia Burgos Jurado

fluido a través de la membrana semipermeable, desde el lado con menor concentración de solutos a un lado con una concentración más alta (Lobo, 2015)

La comprensión de la ósmosis y presión osmótica es importante reconocer los efectos de las diferencias en las cantidades de soluto dentro de la administración de líquidos durante la terapia IV. Debido a que los fluidos que se administran contendrán diferentes cantidades de solutos, su uso tendrá diferentes efectos sobre la ósmosis y presión osmótica dentro de los espacios intravascular y intersticiales.

\section{La reposición de líquidos}

Los expansores de volumen también se pueden utilizar como parte de la reposición de líquidos para el paciente que tiene fluidos o desequilibrios de electrolitos. Algunos pacientes pueden necesitar un reemplazo de líquidos si tienen bajo volumen de líquido, ya que son incapaces de comer o beber líquidos que normalmente reemplazar algunos de sus fluidos. Por ejemplo, los pacientes que han tenido cirugía y no está despierto puede pasar por alto teniendo en fluidos por vía oral durante un largo período de tiempo, y están sujetos a la pérdida de líquidos a través del proceso de la cirugía. Los pacientes quirúrgicos necesitarán reposición de líquidos a través de la terapia IV (Sterns, 2017)Otras situaciones que podrían indicar la necesidad de reemplazo de líquidos incluyen NPO o nada por el estado de la boca durante la hospitalización, las preparaciones intestinales, pérdida insensible de agua, el uso de algunos tipos de medicamentos, y el estrés.

Es responsabilidad del médico de salud para determinar la causa de la hipovolemia con el fin de decidir qué tipo de reposición de líquidos es necesario. Algunos pacientes tienen bajo 


\section{Terapia intravenosa: cristaloides y soluciones de coloides}

Vol. 3, núm. 3., (2019)

Shirley Mercedes Vite Correa; Lizandro Nicolás Anzules Cuzco; Jimmy Eduardo Carreño Ramos; Mónica Cecilia Burgos Jurado

volumen de sangre en el espacio intravascular y el espacio intersticial también se agota. Esto podría ocurrir en tales casos como una gran cantidad de pérdida de sangre a través de trauma, un estado de la cetoacidosis diabética, o deshidratación. Por otra parte, algunos pacientes tienen hipovolemia en el espacio intravascular, pero el espacio intersticial no se agote. Quemaduras o sepsis son condiciones en las que esto podría ocurrir. El tipo y la cantidad de fluido IV dado depende de la condición subyacente del paciente que requiere el reemplazo de fluidos para corregir la hipovolemia.

Los clínicos de salud tienen opciones en cuanto al tipo de fluidos que pueden utilizar para la sustitución de los volúmenes de líquidos y la expansión de volumen. Los tipos de fluidos disponibles típicamente se clasifican como coloides o cristaloides.

\section{Terapia intravenosa coloide}

Los coloides son tipos de soluciones intravenosas que se utilizan para mantener una cantidad adecuada de volumen en el sistema circulatorio, así como la presión dentro de los vasos sanguíneos. Normalmente, el cuerpo produce sustancias dentro de los vasos sanguíneos que mantener el volumen y la presión, que es generalmente el trabajo de la albúmina, fibrinógeno, y globulina. Si estas sustancias no están funcionando correctamente, o si el paciente ha perdido una cantidad significativa de sangre o fluidos corporales, fluidos IV en forma de coloides pueden ser necesarios. Los coloides pueden ser compuestos naturales o sintéticos. Coloides naturales incluyen sangre entera, plasma o de glóbulos rojos. Compuestos de coloides sintéticos son aquellos que se crean con propiedades similares a los fluidos de coloides naturales y que tienen el mismo propósito. 


\section{Terapia intravenosa: cristaloides y soluciones de coloides}

Vol. 3, núm. 3., (2019)

Shirley Mercedes Vite Correa; Lizandro Nicolás Anzules Cuzco; Jimmy Eduardo Carreño Ramos; Mónica Cecilia Burgos Jurado

\section{Características de los coloides}

Coloides ayudan a mantener el volumen intravascular, ya que contienen proteínas que aumentan la presión oncótica dentro de los vasos. la presión oncótica coloide se refiere a la presión ejercida a partir de las partículas de proteína en el torrente sanguíneo contra las paredes de los vasos. Estas partículas son tan grandes que no pasan a través de las membranas semipermeables entre las células y el espacio intravascular. Debido a esto, permanecen en el espacio intravascular para proporcionar volumen. Pueden permanecer en los vasos sanguíneos durante mucho tiempo, lo que permite que sus efectos duran más que las soluciones cristaloides. Además, cantidades menores de soluciones coloides producirán un efecto deseado en un paciente cuando se compara con los cristaloides (Hester, 2013)

\section{El volumen intravascular}

Los coloides son capaces de aumentar el volumen intravascular porque permanecen dentro del espacio intravascular debido al tamaño de sus proteínas y almidones. Ellos también extraen líquido en el espacio intravascular de los espacios intersticiales debido a su presión oncótica. Los coloides son útiles para la expansión de volumen de fluido mediante la utilización de una menor cantidad de total de fluido. Por lo tanto, pueden ser más apropiados para pacientes que no pueden tolerar una gran cantidad de infusión de líquidos, como por ejemplo a través de varias infusiones de soluciones cristaloides. Además, los coloides se pueden administrar a pacientes que están desnutridos o que necesitan proteína adicional debido a que ya contienen proteínas como parte de su maquillaje.

Tipo de coloide 


\section{Terapia intravenosa: cristaloides y soluciones de coloides}

Vol. 3, núm. 3., (2019)

Shirley Mercedes Vite Correa; Lizandro Nicolás Anzules Cuzco; Jimmy Eduardo Carreño Ramos; Mónica Cecilia Burgos Jurado

Dependiendo del tipo de coloide usado, se pueden aplicar ciertos principios de la transfusión. Si un coloide es partir de un compuesto natural o humano, tal como plasma fresco congelado (FFP) o glóbulos rojos empaquetados, precauciones deben ser en su lugar cuando la administración de estos fluidos para monitorizar reacciones de transfusión. Otros tipos de coloides son compuestos sintéticos y mientras que no necesariamente requieren el seguimiento de las reacciones de transfusión, todavía aumentan el volumen intravascular y el médico debe supervisar para detectar signos de sobrecorrección de déficit de volumen de líquido, lo que podría conducir a la hipervolemia. Ya sea sintéticos o naturales, soluciones coloides expanden con éxito el volumen de líquido en el espacio intravascular para corregir el déficit.

\section{Hidroxietil almidon}

También se llama Hetastarch, hidroxietil almidón es un fluido hecho a partir de componentes sintéticos y se utiliza como una infusión de coloide para aumentar el volumen de plasma. (Drugs, 2014) Un ejemplo de almidón de hidroxietilo es Hespan. A pesar de su uso exitoso como expansor de volumen en pacientes con hipovolemia, la Administración de Alimentos y Fármacos de Estados Unidos emitió una medida de seguridad en 2013 para el uso de hidroxietil almidón, que establece que no puede haber un mayor riesgo de mortalidad con su uso en algunos pacientes, incluyendo aquellos con sepsis o fracaso renal racaso. Hidroxietil almidón puede causar daño a los riñones o aumentar el riesgo de hemorragia en estos pacientes.

Alternativamente, hidroxietil almidón puede reemplazar constantemente el volumen entre los pacientes que tienen una pérdida significativa, como entre los pacientes que han tenido una cirugía mayor que requiere una cantidad considerable de succión o donde hubo pérdida 


\section{Terapia intravenosa: cristaloides y soluciones de coloides}

Vol. 3, núm. 3., (2019)

Shirley Mercedes Vite Correa; Lizandro Nicolás Anzules Cuzco; Jimmy Eduardo Carreño Ramos; Mónica Cecilia Burgos Jurado

significativa de sangre. Hidroxietil almidón también se puede usar para la reanimación de volumen para pacientes con quemaduras. Cuando es necesario el trabajo de laboratorio que rodea a la atención al paciente, hidroxietil almidón no interfiere con los resultados de muchos tipos de estudios de laboratorio, aunque algunas otras soluciones coloidales hacen.

\section{Gelofusine}

Gelofusine es un tipo de fluido coloidal que se utiliza como un expansor del volumen; se considera un sustituto del plasma y se utiliza en los pacientes que han experimentado hipovolemia como consecuencia de una hemorragia grave o pérdida de líquidos debido a quemaduras. Gelofusine contiene una mezcla de $4 \%$ succinilada gelatina, sodio, y cloruro. La gelatina modificada funciona de una manera similar a la del plasma. Idealmente se utiliza para personas que necesitan reemplazo de volumen de líquido, pero que por diversas razones no pueden tolerar los productos sanguíneos. Por esta razón, hay menos riesgo de reacción a la transfusión, a pesar de que no se puede utilizar en pacientes que tienen alergia a los productos de gelatina o de huevos.

La administración de Gelofusine requiere una vigilancia estricta para evitar los efectos negativos de la expansión de volumen demasiado, o hipervolemia. Se administra normalmente sólo en situaciones que requieren una vigilancia estrecha del paciente, como en el período perioperatorio o de cuidados intensivos.

La terapia intravenosa con cristaloides 


\section{Terapia intravenosa: cristaloides y soluciones de coloides}

Vol. 3, núm. 3., (2019)

Shirley Mercedes Vite Correa; Lizandro Nicolás Anzules Cuzco; Jimmy Eduardo Carreño Ramos; Mónica Cecilia Burgos Jurado

Cristaloides son fluidos utilizados tanto para expandir el volumen de líquido en el espacio intravascular, así como para proporcionar fluidos de mantenimiento. Pueden ser utilizados para el tratamiento de la hipovolemia y como expansores de volumen; Del mismo modo, también protegen contra otras medidas que pueden conducir a la hipovolemia, como la pérdida insensible de agua o disminución de la ingesta oral de líquidos. Algunos cristaloides contienen electrolitos, por lo que también se pueden utilizar para corregir trastornos electrolíticos.

\section{Características de los cristaloides}

Cristaloides se pueden mezclar con otros líquidos o pueden ser disueltos dentro de otras soluciones. Sus solutos contener partículas suficientemente pequeñas que pueden pasar a través de membranas semipermeables, por lo que tienen la capacidad de afectar el volumen de líquido en el intravascular o espacios intersticiales. Soluciones cristaloides pueden ser clasificadas de manera diferente, dependiendo de su concentración de solutos.

\section{El volumen intravascular}

Las soluciones cristaloides isotónicas son aquellas que no causan un cambio de fluido entre los espacios intracelular y extracelular. Una solución isotónica es la que tiene aproximadamente la misma concentración de solutos como plasma. La presión osmótica es la misma dentro de la célula, ya que es fuera de la célula, por lo que el fluido no se desplaza entre los dos espacios. Debido a que el fluido no se desplaza entre los espacios, soluciones 


\section{Terapia intravenosa: cristaloides y soluciones de coloides}

Vol. 3, núm. 3., (2019)

Shirley Mercedes Vite Correa; Lizandro Nicolás Anzules Cuzco; Jimmy Eduardo Carreño Ramos; Mónica Cecilia Burgos Jurado

isotónicas son útiles para la corrección de déficits de volumen de fluido y la hipovolemia.

Solución salina normal $(0,9 \% \mathrm{NaCl})$ es un ejemplo de una solución isotónica (Marino, 2013)

\section{El volumen intersticial}

Soluciones cristaloides hipertónicas son los que tienen mayores niveles de osmolalidad y las concentraciones más altas de solutos, en comparación con plasma. Cuando se administra por vía intravenosa, soluciones hipertónicas permanecen en el espacio intravascular y tire de fluido fuera del espacio intracelular. Esta atracción de fluido a continuación, aumenta el volumen intersticial, así como corrige bajo volumen de sangre en el espacio intravascular.

Las soluciones hipertónicas contienen electrolitos sodio y cloruro, y algunos tipos pueden contener dextrosa. Son útiles para el tratamiento de los desequilibrios de sodio en los pacientes con hiponatremia debido a su contenido de sodio es mayor que la que ya se encuentra en el torrente sanguíneo. Los pacientes que tienen hipoglucemia grave, también pueden recibir soluciones cristaloides hipertónicas que contienen dextrosa, como la dextrosa adicional está en una concentración mayor que la encontrada en el espacio intravascular. Ejemplos de soluciones hipertónicas incluyen cloruro de sodio 3\% o D10W.

\section{El volumen intracelular}

Fluidos que tienen un nivel inferior de osmolalidad y un menor número de solutos que soluciones isotónicas son conocidos como soluciones hipotónicas. Cuando se dan soluciones hipotónicas, entran en el espacio intravascular, ya que se administran a través de un sitio IV. Debido a su baja osmolalidad, sin embargo, el fluido se desplaza desde el espacio intravascular a 


\section{Terapia intravenosa: cristaloides y soluciones de coloides}

Vol. 3, núm. 3., (2019)

Shirley Mercedes Vite Correa; Lizandro Nicolás Anzules Cuzco; Jimmy Eduardo Carreño Ramos; Mónica Cecilia Burgos Jurado

los espacios intracelulares. El fluido también puede cambiar a los espacios intersticiales. Las soluciones hipotónicas no son útiles para la corrección de déficit de volumen de líquidos porque el líquido no se queda dentro del espacio intravascular. Debido a que tienden a fluir en espacios intracelulares, pueden entonces proporcionar hidratación y aumentar el volumen de fluido celular.

Las soluciones hipotónicas mantener el volumen de fluido celular y son útiles para los líquidos de mantenimiento entre los pacientes. Los únicos electrolitos lo general, contienen son sodio y cloruro; que no son fuentes de otros tipos de electrolitos para corregir los desequilibrios. Las soluciones hipotónicas se utilizan para los pacientes que necesitan el exceso de líquido dentro de las células, como los pacientes con cetoacidosis diabética. Sin embargo, si un paciente se presenta con hipovolemia, la administración de soluciones hipotónicas puede empeorar la condición, por lo que debe administrarse con cuidado u otra solución debe tomar su lugar. Ejemplos de soluciones hipotónicas incluyen cloruro de $0,45 \%$ de sodio $(0,45 \% \mathrm{NaCl})$ y cloruro de sodio $0,2 \%(0,2 \% \mathrm{NaCl})$.

En comparación con el costo de Coloides

Soluciones cristaloides son mucho menos caros de usar en comparación con las soluciones coloidales. No contienen las proteínas que se encuentran en coloides y se realizan en un proceso que es mucho más fácil y más barato para procesar. Además, las soluciones cristaloides no están hechas a partir de productos naturales o hechos por el hombre, en comparación a los coloides, tales como FFP o glóbulos rojos empaquetados, por lo que no requieren donación o recolección de fluidos a producir. En su lugar, una proporción significativa 


\section{Terapia intravenosa: cristaloides y soluciones de coloides}

Vol. 3, núm. 3., (2019)

Shirley Mercedes Vite Correa; Lizandro Nicolás Anzules Cuzco; Jimmy Eduardo Carreño Ramos; Mónica Cecilia Burgos Jurado

de su contenido es agua, que es mucho menos costoso utilizar (Hester, Comparison of IV fluids., 2013).

Tipos de soluciones cristaloides

Hay varios tipos de soluciones cristaloides disponible. A menudo se clasifican de acuerdo a su tonicidad, como se describe anteriormente. La tonicidad se refiere a la medida de la presión osmótica dentro de una solución, y caracterizar una solución como ser isotónica, hipertónica o hipotónica.

\section{Solución salina normal}

La solución salina normal ( $0,9 \%$ de cloruro de sodio) es un tipo de líquido isotónico que cuando se administra, ayuda a aumentar el volumen intravascular. Debido a que es isotónica, solución salina normal no se mueve dentro o fuera de las células, sino que se queda en los vasos sanguíneos en los que se infundió. Solución salina normal contiene agua, sodio y cloruro, y es similar en la concentración de sodio como lo que ya se encuentran en el espacio intravascular. Se utiliza como un expansor de volumen para los pacientes con hipovolemia; y, también puede solicitarse para otros pacientes que necesitan un volumen extra en el espacio extracelular, incluyendo aquellos con shock o acidosis metabólica.

\section{Solución de Ringer lactato}

Similar a lactato de Ringer (LR), solución de Ringer contiene concentraciones del electrolito sodio, potasio, calcio y cloruro en medidas similares a la del plasma sanguíneo. También es una solución isotónica que no se mueve de fluido entre el espacio intracelular y de 


\section{Terapia intravenosa: cristaloides y soluciones de coloides}

Vol. 3, núm. 3., (2019)

Shirley Mercedes Vite Correa; Lizandro Nicolás Anzules Cuzco; Jimmy Eduardo Carreño Ramos; Mónica Cecilia Burgos Jurado

los vasos, lo que es ideal para el reemplazo de volumen de líquido. Solución de Ringer y LR se pueden utilizar en los pacientes para los esfuerzos de reanimación con líquidos y durante la terapia de mantenimiento de líquidos durante la cirugía. Solución de Ringer lactato no contiene que se encuentra en LR, por lo que no se utiliza en los pacientes con acidosis láctica. Sin embargo, se puede usar entre una variedad de otros pacientes que se presentan con condiciones tales como quemaduras o deshidratación.

D5W

D5W es una combinación de 5\% de dextrosa en agua. Es un fluido que se clasifica ya sea como una solución isotónica o hipotónica. Es isotónica en que la cantidad de dextrosa contenida en el líquido es similar a la encontrada en los vasos sanguíneos. Sin embargo, el cuerpo utiliza rápidamente la dextrosa se encuentra en D5W y así el agua restante puede pasar a través de las membranas semipermeables y entrar en los espacios intersticiales e intracelulares. D5W es útil para proporcionar calorías, pero que no contiene electrolitos, por lo que no es útil para corregir los desequilibrios de electrolitos. Está también normalmente no se utiliza como parte de la reanimación con líquidos o para corregir la hipovolemia severa porque el líquido no permanece en el espacio intravascular. En cambio, puede ser utilizado como una forma de líquido de mantenimiento, aunque la dextrosa al 5\% no es suficiente para calorías o nutrición a largo plazo.

\section{Conclusiones.}

La terapia intravenosa implica la administración de fluidos, medicamentos, nutrientes y electrolitos a través de las venas. Clínicos de salud que administran la terapia intravenosa deben 


\section{Terapia intravenosa: cristaloides y soluciones de coloides}

Vol. 3, núm. 3., (2019)

Shirley Mercedes Vite Correa; Lizandro Nicolás Anzules Cuzco; Jimmy Eduardo Carreño Ramos; Mónica Cecilia Burgos Jurado

entender los principios básicos de la corrección de los desequilibrios de electrolitos, ampliando el volumen de líquido en el sistema circulatorio, y la reposición de líquidos durante la enfermedad o lesión física. El tipo de fluido intravenoso se selecciona basándose en el estado y los requisitos para mantener el equilibrio de líquidos correcto física del paciente. La terapia intravenosa es un componente común e importante de la atención clínica. Cuando los pacientes presentan necesidades del equilibrio de líquidos y electrolitos debido a una cirugía, lesión o enfermedad, es responsabilidad de un clínico experto colaborar con todos los miembros interdisciplinarios del equipo de salud sobre el más apropiado tipo de solución intravenosa y velocidad de infusión a administrar.

\section{Bibliografía.}

Bachur, R., \& Shaw, K. (2015). Fleisher \& Ludwig's Textbook of Pediatric Emergency Medicine. Philadelphia, PA: Lippincott Williams \& Wilkins.

Drugs. (2014). Hetastarc. Obtenido de http://www.drugs.com/pro/hetastarch.html

Hester, S. (2013). Comparison of IV fluids. Obtenido de http://portal.mah.harvard.edu/cms/content/B7ACBE692D3340DD9CD3 08883BC9750B/1E30C939CCAF446D8D27DD0F639C15E7.pdf

Hester, S. (2013). Comparison of IV fluids. Obtenido de http://portal.mah.harvard.edu/cms/content/B7ACBE692D3340DD9CD3 08883BC9750B/1E30C939CCAF446D8D27DD0F639C15E7.pdf

Lobo, D. (2015). Basic concepts of fluid and electrolyte therapy. Obtenido de http://www.bbraun.com/documents/Knowledge/Basic_Concepts_of_Flui d_and_Electrolyte_Therapy.pdf

Marino, P. (2013). Marino's the ICU book. Philadelphia,: Lippincott Williams \& Wilkins.

Moya, M. (2013). Intravenous fluid resuscitation. Obtenido de http://www.merckmanuals.com/professional/critical_care_medicine/sho ck_and_fluid_resuscitation/intravenous_fluid_resuscitation.html 


\section{Terapia intravenosa: cristaloides y soluciones de coloides}

Vol. 3, núm. 3., (2019)

Shirley Mercedes Vite Correa; Lizandro Nicolás Anzules Cuzco; Jimmy Eduardo Carreño Ramos; Mónica Cecilia Burgos Jurado

Siparsky, N. (2017). Overview of postoperative fluid therapy in adults. Obtenido de https://www.uptodate.com/contents/overview-of-postoperative-fluid-therapy-inadults?source $=$ search_result $\&$ search $=$ volume $\% 20$ expanders \&selectedTi tle $=2 \sim 15$

Sterns, R. (2017). Etiology, clinical manifestations, and diagnosis of volume depletion in adults. UpToDate. Obtenido de https://www.uptodate.com/contents/etiology-clinicalmanifestations-and-diagnosis-of-volume-depletion-inadults?source=search_result\&search=intravenous $\% 20$ volume $\% 20$ expa nders\&selectedTitle $=1 \sim 150$. 